\title{
Contraste de un modelo de los determinantes de la estancia turística en la era covid-19: implicaciones para la bioseguridad
}

\author{
Contrast of a model of the determinants of tourist stay in \\ the covid-19 era: implications for biosafety
}

\author{
Cruz García Lirios \\ Universidad Autónoma del Estado de México \\ Juan Antonio Garza Sánchez* \\ Universidad Autónoma de Nuevo León, México \\ Tirso Javier Hernández Gracia \\ Universidad Autónoma del Estado de Hidalgo, México \\ Javier Carreón Guillén \\ Universidad Nacional Autónoma de México, México \\ Francisco Espinoza Morales \\ Universidad de Sonora, México
}

\section{RESUMEN}

La reactivación del turismo se venía estudiando por la influencia del habitus de traslado y estancia, pero con la contingencia sanitaria este proceso estuvo mediatizado por la información de los lugares con protocolos de bioseguridad. El objetivo de esta investigación es contrastar un modelo de los determinantes de la estancia, considerando las variables mediadoras de la información. Es un estudio psicométrico y transversal que se llevó a cabo con una selección de 100 estudiantes de una universidad pública en el centro de México. Se encontró una estructura factorial que explica el $61 \%$ de la varianza y establece la intención de transferir como predictor, sugiriendo la extensión del trabajo hacia protocolos de bioseguridad.

Palabras clave: globalización, percepción de utilidad y uso de Internet móvil. 


\begin{abstract}
The reactivation of tourism had been studied by the influence of the habitus of movement and stay, but with the health contingency, this process was mediated by the information from the places with biosecurity protocols. The objective of the present work was to contrast a model of the determinants of stay, considering the mediating variables of the information. A confirmatory, psychometric, and cross-sectional study was carried out with a selection of 100 students from a public university in central Mexico. A factorial structure was found that explained $61 \%$ of the variance and established the intention to transfer as a predictor, suggesting the extension of the work towards biosafety protocols.
\end{abstract}

Keywords: globalization, perception of utility and use of mobile Internet.

\title{
Introducción
}

En la actualidad la COVID-19, enfermedad causada por el nuevo coronavirus SARS-CoV-2 viene causando la muerte de más de dos millones de personas en el mundo. En México las muertes superan los 150 000, pero se reconoce un subregistro que podría elevar la cifra a 500 000. En este escenario de alto riesgo, las políticas para mitigar y contener la pandemia se han centrado en el encierro y el distanciamiento social, conduciendo a la recesión económica y al desempleo, pero también al emprendimiento, que se ha intensificado y diversificado desde protocolos de bioseguridad para micro, pequeñas y medianas empresas (Organización Mundial de la Salud [OMS], 2021). De esta forma, bioseguridad y emprendimiento se cruzan en un escenario de alto riesgo de contagio, enfermedad y muerte, ya que en México la tasa de letalidad es de 10.2\%, superior a cualquiera de las economías de la Organización para la Cooperación y el Desarrollo Económicos (OCDE).

En un entorno turístico, la bioseguridad es asumida como la prevención de riesgos y enfermedades asociada a la ejecución de una tarea o función laboral, recreativa o académica. En la era COVID-19, el protocolo de bioseguridad incluye el uso obligatorio de mascarillas, medición de $\mathrm{CO} 2$, aplicación de pruebas de detección PCR, dosis de vacunación, distanciamiento social y aislamiento (González, 2020).

El emprendimiento es un eje central de la reactivación económica en general y del turismo (Bustos et al., 2021). La relación entre transporte y destinos vacacionales configura una red de emprendimiento en la que las promociones son el factor determinante del turismo y se espera que sean el factor desencadenante de su reactivación (García et al., 2019). Es decir, la experiencia del traslado o la satisfacción del viaje se relacionan positiva y significativamente con la experiencia o satisfacción con la estadía, así como con los valores agregados de las visitas a áreas culturales y recreativas. 
Se trata de una red de traslado y estancia que se estructura en habitus turísticos; estética, ética, conocimiento y expresividad (García et al., 2018). Estos cuatro ejes explican la inteligencia empresarial de aerolíneas y hoteles para ofrecer un destino basándose en las cuatro experiencias satisfactorias de traslado y estadía.

Sin embargo, con el surgimiento de la pandemia, la red de conocimiento turístico se ha modificado desde la indefensión al reestructurarse en experiencias libertarias frente el encierro, así como en experiencias compensatorias ante el posmaterialismo que implica el distanciamiento social y económico (García et al., 2020). Esta es la forma en la inteligencia del negocio de turismo; pasó de ser experiencias hedonistas a experiencias eudamónicas (Carreón et al., 2019). Es decir, el placer de viajar, descansar y conocer fue reemplazado por el autocuidado y la responsabilidad con el medioambiente.

A diferencia del habitus hedonista que consiste en la satisfacción personal sin considerar el entorno inmediato, los habitus eudamónicos son satisfacciones prolongadas de identidad y seguridad con el entorno (Carreón et al., 2019). Antes, los turistas buscaban recreación y entretenimiento y ahora salen del encierro, ajustando sus acciones a los protocolos de bioseguridad.

En México, un gran sector de turistas contrajo la infección en las congregaciones, pero acudiendo a los centros de salud o siguiendo los protocolos de salvaguarda han evitado complicaciones. De hecho, la mayoría de las víctimas son personas que no realizaron un viaje de ocio en el último año o estuvieron expuestas en zonas y colectivos de alto riesgo como el comercio informal.

La red de traslados y estadías, al estar determinada por la necesidad y búsqueda de información, sugiere un proceso útil de las páginas de aerolíneas, hoteles y destinos turísticos, así como una eficiencia a la hora de encontrar ofertas en fechas previstas (Carreón et al., 2019). En consecuencia, la utilidad y eficiencia en el uso de la información turística determina la decisión de buscar ofertas y promociones, así como la compra de paquetes y el consiguiente traslado y estadía al área seleccionada.

De esta forma, el habitus estético que consiste en la experiencia satisfactoria de apreciar el entorno estaría ligado a la percepción de utilidad a la hora de comprar una reserva (Carreón et al., 2019). O bien, la ética del consumo estaría asociada a la eficiencia de encontrar la oferta menos contaminante. Son estas relaciones las que explican la reactivación del turismo basado en un proceso de turismo ético, estético, racional, deliberado, planificado, sistemático y expresivo.

El objetivo de esta investigación es contrastar un modelo de los determinantes de la experiencia de traslado y estadía basado en el habitus estético, ético, racional y expresivo, así como la percepción de utilidad y eficiencia en la búsqueda de ofertas de viaje y estadía.

¿Existen diferencias entre la estructura de determinantes de la experiencia de reserva, traslado y estadía 
reportada en la literatura con respecto a la estructura observada en el presente trabajo?

La premisa que sustenta esta investigación asume que la pandemia modificó las relaciones entre el habitus y la experiencia de traslado y estadía, desplazándolos por la necesidad y eficiencia del procesamiento de la información al momento de planificar unas vacaciones (Juárez et al., 2021). En ese sentido, la utilidad percibida y la eficiencia de la gestión de datos determinan la satisfacción del cliente independientemente de su exposición al contagio mientras se desplaza o permanece en un hotel cerrado o abierto para ventilación y distanciamiento (Sandoval et al., 2021).

\section{Método}

\section{Diseño}

El estudio es transversal y psicométrico con una selección de internautas, considerando su tiempo de uso. En la primera fase, se construyó y estableció la confiabilidad y validez de los instrumentos que midieron las cinco variables. En la segunda fase, se modeló y demostró la probabilidad de ajustar relaciones causales indirectas y directas, negativas y positivas, y significativas entre las variables de estudio.

\section{Muestra}

Para obtener la muestra se realizó una selección probabilística de 100 estudiantes de una universidad pública del centro de México, considerando sus prácticas profesionales en los servicios turísticos, servicio social en instituciones de gestión del turismo y asistencia a eventos internacionales como congresos. Se utilizó la siguiente fórmula: $\mathrm{m}=\mathrm{N} /(\mathrm{N}-1) * \mathrm{~K} 2+1$

Siendo m la muestra, N la población y K el margen de error con el 5\% (López, 2004, p. 70).

\section{Instrumento}

Como instrumento se seleccionó la Escala de Reactivación Turística de García et al. (2020), que incluye nueve variables: (1) habitus estético (Visitar lugares atractivos); (2) ético (Protocolo de respeto a la bioseguridad); (3) expresivo (Visitar lugares entrañables); (4) racional (Visitar lugares con semáforos verdes); (5) percepciones de utilidad (En esta página encontraré una oferta de semáforo verde); (6) facilidad (En esta página encontraré rápidamente una oferta de viaje y alojamiento); (7) en la búsqueda de información, que determinan las variables de intención de reserva (Reservaría en esta página si estuviéramos en un semáforo en verde); (8) transferencia (Viajaría en esta aerolínea si estuviéramos en un semáforo en verde); y (9) quedarse (Me quedaría en este hotel si tuviéramos luz verde). Todos incluyen cinco opciones de respuesta que van desde $0=$ poco probable a 5 = bastante probable. 


\section{Proceso}

Las propiedades psicométricas de los instrumentos que miden las variables de estudio se detallan en la Tabla 1, donde se cumplen los requisitos para el análisis multivariable. Durante la primera semana del trimestre de primavera de 2020, en la página de la universidad pública y con el objetivo de seleccionar la muestra ideal, se preguntó a los estudiantes con qué frecuencia usaban su teléfono para descargar imágenes, sonidos y discursos. Posteriormente, se envió el cuestionario indicando un tiempo de respuesta de 30 minutos para contestarlo.

\section{Análisis}

Se estimaron los parámetros de confiabilidad, validez, adecuación, esfericidad, correlación, covarianza, ajuste y residual (Hernández et al., 2019). Se asumieron como relaciones espurias valores cercanos a cero a excepción del parámetro residual, que se consideró como una clarividencia de contraste de la hipótesis nula (Martínez et al., 2019). Además, se distinguieron valores cercanos a la unidad como evidencia de no rechazo de la hipótesis nula (Villegas, 2019).

\section{Resultados}

En la Tabla 1 se muestran los valores de confiabilidad y validez que sugieren una estructura factorial de ejes principales con rotación promax. En otras palabras, las variables parecen configurar un proceso híbrido de planificación e improvisación a la hora de tomar la decisión y ejecutar la intención de viajar y permanecer en un destino turístico.

\section{Tabla 1}

Instrumento descriptivo

\begin{tabular}{|c|c|c|c|c|c|c|c|c|c|c|c|c|}
\hline$R$ & $M$ & $D E$ & $A$ & $F 1$ & $F 2$ & $F 3$ & $F 4$ & $F 5$ & F6 & $F 7$ & $F 8$ & $F 9$ \\
\hline$r l$ & 4.1 & 1.1 & .71 & .32 & & & & & & & & \\
\hline$r 2$ & 4.3 & 1.2 & .72 & .43 & & & & & & & & \\
\hline$r 3$ & 4.5 & 1.3 & .78 & .46 & & & & & & & & \\
\hline r4 & 4.6 & 1.0 & .70 & & .54 & & & & & & & \\
\hline$r 5$ & 4.4 & 1.1 & .77 & & .61 & & & & & & & \\
\hline r6 & 4.1 & 1.8 & .74 & & .68 & & & & & & & \\
\hline$r 7$ & 4.0 & 1.9 & .72 & & & .54 & & & & & & \\
\hline$r 8$ & 4.4 & 1.4 & .77 & & & .58 & & & & & & \\
\hline r9 & 4.6 & 1.7 & .79 & & & .52 & & & & & & \\
\hline r10 & 4.8 & 1.3 & .70 & & & & .43 & & & & & \\
\hline$r 11$ & 4.9 & 1.6 & .74 & & & & .47 & & & & & \\
\hline$r 12$ & 4.4 & 1.5 & .72 & & & & .58 & & & & & \\
\hline$r 13$ & 4.3 & 1.2 & .71 & & & & & .65 & & & & \\
\hline rl4 & 4.1 & 1.3 & .77 & & & & & .68 & & & & \\
\hline
\end{tabular}




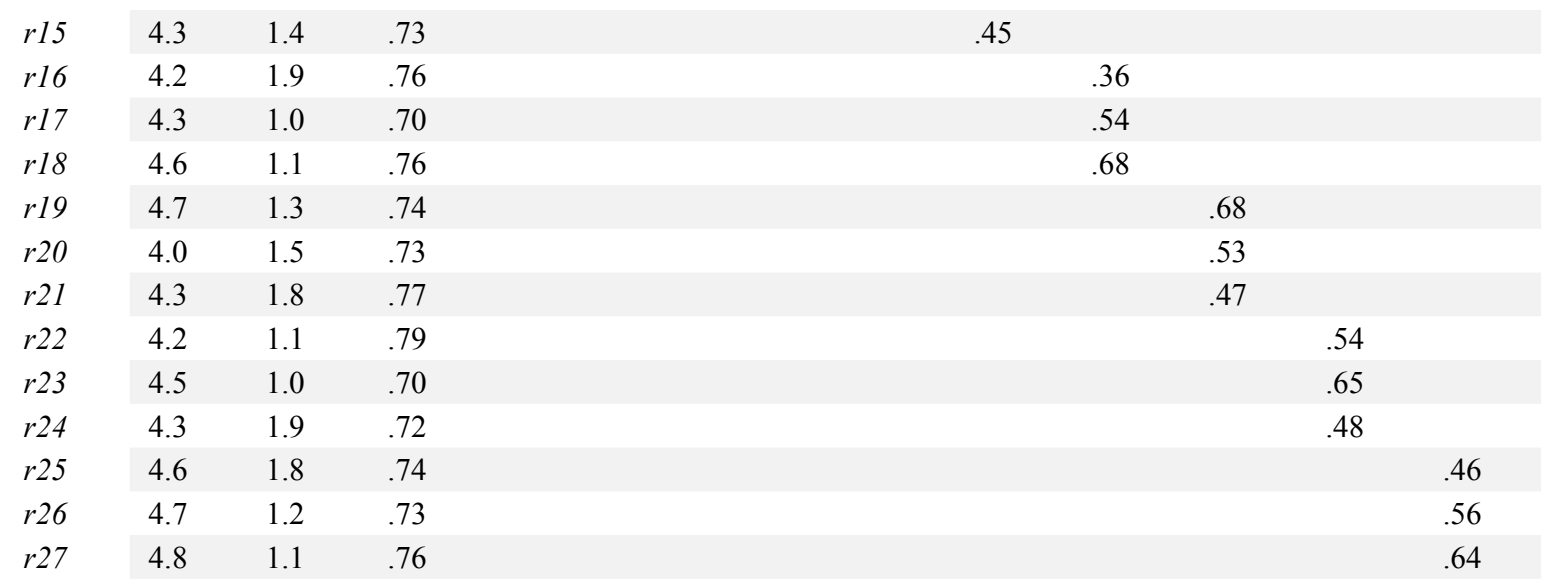

Nota. Elaborado con estudio de datos; Adecuación $(\mathrm{KMO}=0.890)$, Esfericidad $\lceil\chi 2=213.24(24 \mathrm{gl}) \mathrm{p}<.05\rfloor$ Extracción: Ejes principales, Rotación: Promax. M = Media, DE = Desviación estándar, A = Ítem de valor excluido alfa, F1 = Aesthesis $(16 \%$ de varianza total explicada y alfa con .780), F2 = Ethos (13\% de varianza total explicada y alfa con, 775$), \mathrm{F} 3=$ Hexis (10\% de varianza total explicada y alfa con .759), F4 = Eidós (7\% de varianza total explicada y alfa con, 771), F5 = Utilidad (5\% de varianza total explicada y alfa con .776), F6 = Facilidad ( 4\% de varianza total explicada y alfa con, 795) .F7 = Reservación (3\% de varianza total explicada y alfa con, 783) $. \mathrm{F} 8=$ Traslado ( $2 \%$ de varianza total explicada y alfa con .762), F9 = Estadía (1\% varianza total explicada y alfa con .750).

La estructura factorial explicó $61 \%$ de la varianza total y sus 10 factores se estimaron por sus relaciones con el fin de ser capaz de apreciar su estructura de asociación (véase la Tabla 2).

\section{Tabla 2}

Modelado de ecuciones estructurales

\begin{tabular}{|c|c|c|c|c|c|c|c|c|c|c|c|}
\hline & $M$ & $\mathrm{DE}$ & $F I$ & $F 2$ & $F 3$ & F4 & $F 5$ & F6 & $F 7$ & $F 8$ & $F 9$ \\
\hline$F 1$ & 23.21 & 15.21 & 1.00 & $.312 *$ & $.334 *$ & $.314 *$ & $.324 * *$ & $.346 *$ & $.435 *$ & $.354 *$ & $.543 *$ \\
\hline$F 2$ & 24.35 & 16.57 & & 1.00 & $.313 *$ & $.354 *$ & $.313 *$ & $.343 *$ & $.421 * *$ & $.657 * *$ & $.643 * *$ \\
\hline$F 3$ & 26.54 & 14.35 & & & 1.00 & $.324 *$ & $.432 *$ & $.531 * *$ & $.672 * * *$ & $.435 * *$ & $.578 *$ \\
\hline F4 & 23.21 & 13.24 & & & & 1.00 & $.321 * * *$ & $.325 *$ & $.543 *$ & $.652 * * *$ & $.435 *$ \\
\hline F5 & 27.68 & 15.46 & & & & & 1.00 & $.324 * *$ & $.453 *$ & $.546 *$ & $.657^{*}$ \\
\hline F6 & 22.34 & 16.58 & & & & & & 1.00 & $.532 * *$ & $.547 *$ & $.642 * * *$ \\
\hline$F 7$ & 25.31 & 15.21 & & & & & & & 1.00 & $.653 * *$ & $.546^{*}$ \\
\hline$F 8$ & 24.35 & 10.23 & & & & & & & & 1.00 & $.432 *$ \\
\hline F9 & 26.54 & 16.28 & & & & & & & & & 1.00 \\
\hline
\end{tabular}

Nota . Elaborado con estudio de datos; $\mathrm{M}=$ Media, DE = desviación estándar, F1 = Aesthesis, F2 = Ethos, F3= Hexis, F4= Eidos, F5 $=$ Utilidad, F6 $=$ Facilidad, F7 $=$ Reservación, F8 $=$ Traslado, F $9=$ Estadía: $* \mathrm{p}<.01 ; * * \mathrm{p}<.001 ; * * \mathrm{p}<.0001$

La estructura de correlación sugiere ejes, trayectorias y relaciones entre las variables determinantes de la intención de reserva, traslado y estadía, corroborando plenamente la estructura de relación parcial reportada por Morillo (2011, p. 143) y Navarro (2015, p. 342) (ver Figura 1). 


\section{Figura 1}

Modelado de ecuaciones estructurales

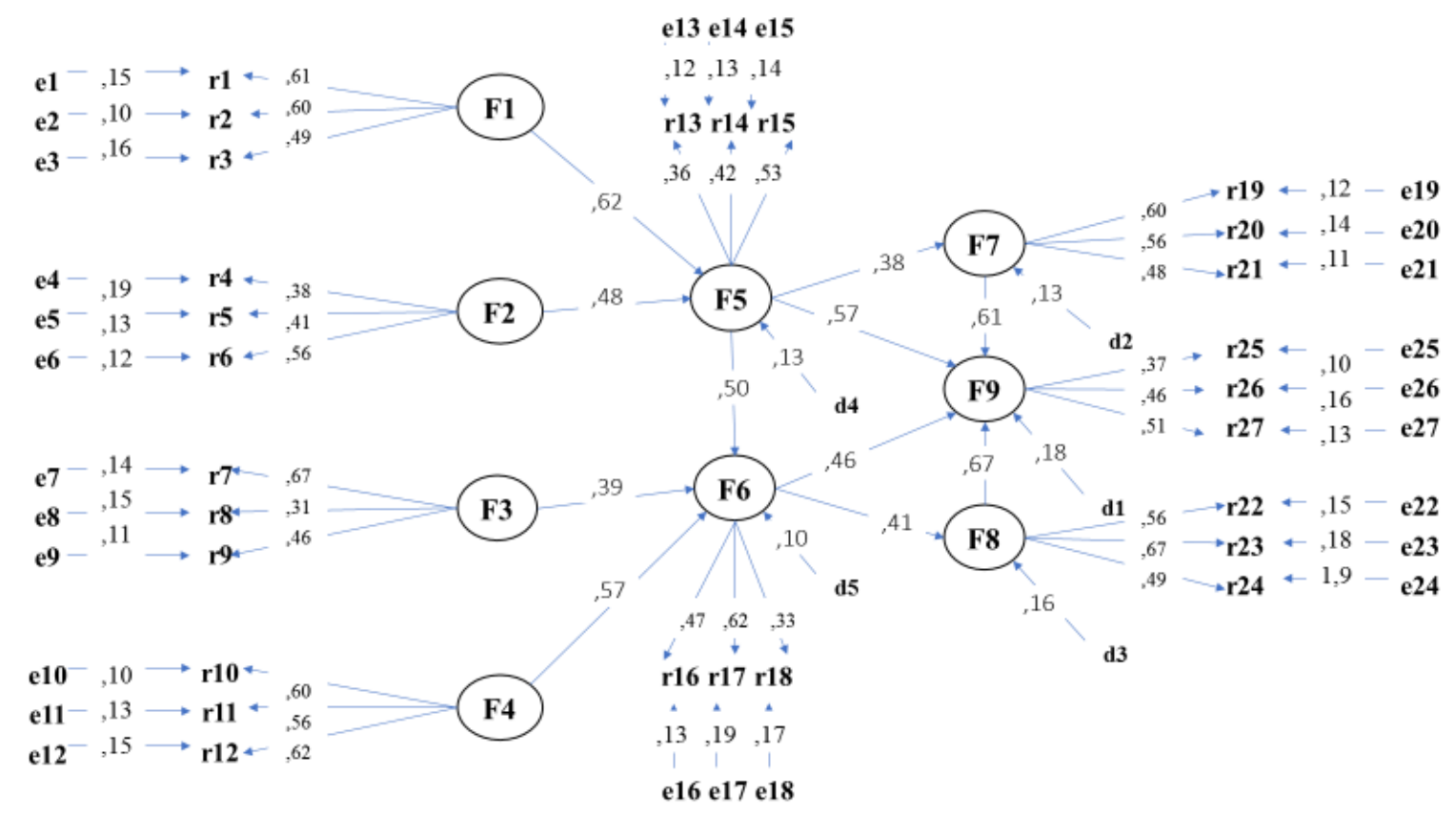

Nota . Elaborado con estudio de datos; F1 = Aesthesis, F2 = Ethos, F3 = Hexis, F4 = Eidos, F5 = Utilidad, F6 = Facilidad, F7 = Reservación, F8 = Traslado, F9 = Estadía; $\mathrm{r}=$ reactivo, e = elemento de medición de error, $\mathrm{d}$ = factor de medición de perturbaciones; $\leftarrow$ relación entre factor e indicador, error e indicador, perturbación y factor; $\rightarrow$ relaciones entre factores.

El modelo de ecuación estructural muestra que el predictor de estancia es la transferencia. Es una estructura de ejes, trayectorias y relaciones entre las variables determinantes de la estancia. A su vez, la facilidad de uso de la información determina la experiencia de transferencia. Es decir, la información disponible en Internet sobre una aerolínea y su vínculo con el alojamiento determina la intención de mudarse. Este proceso comienza con el habitus de expresividad que afecta la facilidad de uso. De hecho, la disposición a favor de un destino turístico favorece la búsqueda de ofertas y la experiencia satisfactoria de viajar a ese lugar.

Los parámetros de ajuste y residuales $\lceil\chi 2=214.34(24 \mathrm{df}) \mathrm{p}>.09$; GFI $=.997$; CFI $=.995$; RMSEA $=$ $.008 \mathrm{~J}$ sugieren el no rechazo de la hipótesis nula que indica diferencias significativas entre la estructura reportada en la literatura consultada con respecto a la estructura observada en el presente estudio.

\section{Discusión}

El aporte de la investigación radica en el contraste de un modelo de los determinantes de la intención de estancia turística sugerida por Vidrio et al. (2020, p. 2). La hipótesis relativa a las diferencias significativas entre los ejes, trayectorias y relaciones establecidas en la literatura no fue rechazada en el presente estudio, ya 
que los parámetros de ajuste sugieren un ajuste de la teoría del modelo con respecto a la prueba empírica del modelo (Escobedo et al., 2016, p. 17). Es decir, en otros escenarios y muestras similares, el modelo propuesto puede servir para ser contrastado con el fin de dotarle de evidencia empírica, así como ajustar las relaciones entre factores e indicadores (Ritzel et al., 2020, p. 10).

Un modelo estructural se fundamenta por el porcentaje de varianza total explicada de sus factores en relación con sus indicadores (Scheweizer et al., 2019, p. 2). En el presente trabajo, la estructura factorial explicó el $61 \%$ de la varianza total, sugiriendo que la intención de moverse determina la intención de quedarse, trayectoria planteada por Campos y Corcho (2019, p. 158). Esto es así porque el habitus de la expresividad explica las razones para visitar lugares significativos para el turista. Además de esta distinción, la facilidad de acceso a las promociones determina el traslado al destino seleccionado.

En relación con la estructura factorial reportada en la literatura y que sugiere la incidencia del habitus sobre la experiencia satisfactoria del traslado y estadía propuestos por Sánchez et al. (2007, p. 34), se corrobora esta trayectoria y sugiere que dicho proceso está mediado por la facilidad de acceso a promociones. Esta pregunta es fundamental para anticiparnos a la reactivación turística siempre que las páginas de las aerolíneas y hoteles sean explícitas en cuanto a promociones ajustadas a la bioseguridad que la situación demanda.

Líneas de investigación sobre el conocimiento y la intención de realizar el protocolo de seguridad; el uso de mascarillas, gel de alcohol, careta, oxímetro y el distanciamiento social explicarían las posibilidades de reactivar el turismo. Es decir, el protocolo de bioseguridad como directriz para las partes involucradas mediará el proceso de identidad y satisfacción que explica el turismo.

\section{Conclusiones}

El objetivo es contrastar el modelo de los determinantes de la estancia turística, considerando una revisión de la literatura sobre habitus y aceptación de las tecnologías de la información. Los resultados sugieren el no rechazo de la hipótesis nula, aludiendo a las diferencias significativas entre la estructura teórica con respecto a la estructura observada. Una línea de investigación derivada del protocolo de bioseguridad para reactivar el turismo sugiere que el uso de mascarilla, gel de alcohol, mascarilla, oxímetro y distancia saludable mediaría el proceso de traslado y estancia.

En cuanto a las políticas públicas relacionadas con la reactivación del turismo en tiempos de pandemia, se sugiere una intensa promoción de los destinos turísticos en las redes sociales, ya que el habitus expresivo se manifiesta a menudo en fotos o videos sobre experiencias de viaje, estadía y retorno. El acceso y la facilidad de búsqueda de información, junto con la experiencia de transferencia, determinarían la elección de un destino turístico. 


\section{Conflicto de intereses}

Los autores no tienen conflicto de intereses con el contenido de este trabajo.

\section{Contribución de autoría}

CGL: Gestión de recursos y revisión de análisis estadísticos y documento final.

JASG: Curación de datos y análisis estadísticos.

TJHG: Elaboración de marco teórico y análisis estadísticos.

JCG: Recolección y curación de datos.

FEM: Revisión de la literatura y elaboración de marco teórico.

\section{Referencias}

Bustos, J. M., García, C. y Juárez, M. (2021). Validez del modelo de habitus de espíritu empresarial. Summa, 3(1), 1-21. https://dx.doi. org/ 10.47666 / summa.3.1.02

Campos, L. M. y Corcho, I. L. (2019). Movilidad estudiantil y turismo académico. Miradas comunes entre la internalización y el turismo. Micarimin, 6, 147-170 http://45.238.216.13/ojs/index.php/mikarimin/article/view/1719/922

Carreón, J., Fierro, E. y García, C. (2019). Modelos de efectos fijos de variables difusas en la formación de capital intelectual. Revista Internacional de Investigación y Desarrollo en Ingeniería, 15(9), 1-7.

Carreón, J., Hernández, T. J. y García, C. (2019). Estructura categorial exploratoria de expectativas laborales. Revista de Investigación en Ciencias Sociales, 6(8), 1-6.

Carreón, J., Villegas, E. y García, C. (2019). Modelo de los determinantes del capital humano. Revista Internacional de Avances en Ciencias Sociales y Humanidades, 7(8), 1-5.

Escobedo, M. T., Hernández, J. A., Estebané, V. y Martínez, G. (2016). Modelos de ecuaciones estructurales: Características, fases, construcción, aplicación y resultados. Ciencias \& Trabajo, 18(55), 16-22. https://scielo.conicyt.cl/pdf/cyt/v18n55/art04.pdf

García, C., Espinoza, F. y Carreón, J. (2018). Modelo de activos intangibles y capitales en organizaciones. Revista Internacional de Investigación en Humanidades y Estudios Sociales, 5(4), 1-12.

García, C., Martínez, E. y Quintero, M. L. (2019). Estructura factorial exploratoria clima y flexibilidad laboral. Vueltas, 20(2), 55-72.

García, C., Quiroz, C. Y., Bermúdez, G., Sandoval, F. R. y Carreón, O. I. (2020). Fiabilidad y validez de un instrumento que mide el emprendimiento. Pensamiento y Gestión, 49, 276-293

González, A. (2020). Evaluación del nivel de conocimiento sobre los protocolos de bioseguridad en el personal del establecimiento de alojamiento, provincia de Tungurangua ante la presencia del SARS CoV-2. Investigación y Desarrollo, 13, 1-10. https:// revistas.uta.edu.ec/erevista/index.php/dide/article/view/1004

Hernández, T. J., Carreón, J. y García, C. (2019). Netizens Millennials. International Journal of Advances Engineering Investigación y Ciencia, 6(7), 1-5.

Juárez, M., Quiroz, C. Y., Bustos, J. M. y García, C. (2021). Validez de la Escala de Mediación en Internet en estudiante de una Universidad Pública Mexicana. Eureka, 18(1), 43-54.

López, L. J. (2004). Población, muestra y muestreo. Punto Cero, 9(8), 69-74. http://www.scielo.org.bo/pdf/rpc/v09n08/v09n08a12.pdf

Martínez, E., Espinoza, F. y García, C. (2019). Modelos de los determinantes de la formación profesional. Revista internacional de avances en ciencias sociales y humanidades, 6(7), 1-5.

Morillo, M. (2011). Turismo y producto turístico. Evolución, conceptos, componentes y clasificación. Visión Gerencial, 1, 135-158. https://www.redalyc.org/pdf/4655/465545890011.pdf

Navarro, D. (2015). Recursos y atractivos turísticos, conceptualización, clasificación y valoración. Cuadernos de Turismo, 35, 335-357. https://dx.doi.org/10.6018/turismo.35.221641 
20 | JUAN ANTONIO GARZA SÁNCHEZ, TIRSO JAVIER HERNÁNDEZ GRACIA, JAVIER CARRIÓN GUILLÉN, FRANCISCO ESPINOZA MORALES, CRUZ GACÍA LIRIOS

Organización Mundial de la Salud (OMS). (2021). Estadísticas del coronavirus SARS CoV- 2 y la enfermedad COVID-19 en el mundo. OMS.

Ritzel, C., Mack, G., Portmann, M., Heitkamper, K. y El Benni, N. (2020). Empirical evidence on factors influencing farmers' administrative burden: A structural equation modeling approach. PLoS ONE, 15(10), e0241075. https://doi.org/10.1371/journal. pone. 0241075

Sánchez, S. M., López, T. J. y Millán, G. (2007). La satisfacción laboral en los establecimientos hoteleros. Análisis empírico de la provincia de Córdova. Cuadernos de Turismo, 20, 223-249. https://www.redalyc.org/pdf/398/39802010.pdf

Sandoval, F., Bustos, J. M., Juárez, M. y García, C. (2021). Especificación de un modelo para el estudio de la sostenibilidad de la obra social. Educación, sociedad y medio ambiente, 8(1), 343-353.

Scheweizer, K., Troche, S. J. y DiStefano, C. (2019). Sacaling the variance of latent variable while assuring constancy of the model. Frontiers in Psychology, 10, 887-897. https://doi.org/10.3389/fpsyg.2019.00887

Vidrio, S, B., Rebolledo, A. R. y Galindo, S. D. (2020). Calidad del servicio hotelero, lealtad e intención de compra. Investigación Administrativa, 49(125), 1-19. http://www.scielo.org.mx/pdf/ia/v49n125/2448-7678-ia-49-125-00002.pdf

Villegas, E. (2019). Gobernanza del capital intelectual millennials para la creación de valores organizacionales intangibles. Net Diario de Ciencias Sociales, 6(1), 1-9.

Juan Antonio Garza Sánchez

Doctor en Ciencias de la Información y máster en Comunicación Periodística Empresarial e Institucional por la Universidad por la Universidad Complutense de Madrid (España). Licenciado en Comunicación por la Universidad Autónoma de Nuevo León (México). Profesor e Investigador de la UANL, cuenta con el reconocimiento al Perfil Deseable del PRODEP y es miembro del Sistema Nacional de Investigadores del CONACYT, Nivel I.

juan.garzasnc@uanl.edu.mx

ORCID: https://orcid.org/0000-0002-8536-6321

Tirso Javier Hernández Gracia

Doctor en Ciencias Administrativas por la Facultad de Contaduría y Administración de la Universidad Autónoma de Tlaxcala (México). Profesor Investigador del Área Académica de Administración en el Instituto de Ciencias Económico Administrativas de la Universidad Autónoma del Estado de Hidalgo (México). Docente de asignaturas como Diseño Organizacional, Fundamentos de Metodología de la Investigación, Estrategias de Dirección de Capital Humano y Metodología de Estudio de Casos, tanto a nivel licenciatura como maestría. Reconocimiento y certificación ante ANFECA.

thernan@uaeh.edu.mx

ORCID http://orcid.org/0000-0003-0425-0800

Javier Carreón Guillén

Doctor en Administración (Organizaciones). Profesor de Carrera Titular "C", Universidad Nacional Autónoma de México, Escuela Nacional de Trabajo Social. Línea de Investigación en Gobernanza del Desarrollo Humano, Adscrito al Sistema Nacional de Investigadores, Consejo Nacional de Ciencia y Tecnología.

javierg@unam.mx

ORCID: http://orcid.org/0000-0002-8915-0958

Francisco Espinoza Morales

Maestro en Administración por la Universidad de Sonora (México). Doctor en Educación por la Universidad de Tijuana (México). Profesor de Tiempo Completo en el Departamento de Ciencias Económico Administrativas y secretario académico de la División de Ciencias Económicas y Sociales de la Unidad Regional Sur de la Universidad de Sonora, miembro del Sistema Nacional de Investigadores (SNI) de CONACYT, reconocimiento por la Secretaría de Educación Pública (SEP) Perfil PRODEP, líder del Cuerpo Académico de Desarrollo Regional y Competitividad, acreditado por la Asociación Nacional de Facultades y Escuelas de Contabilidad y Administración (ANFECA).

fespinoz@navojoa.uson.mx

ORCID: https://orcid.org/0000-0002-4552-5893

Cruz García Lirios

Posdoctorante en Gobernanza de la Complejidad. Profesor de asignatura, Universidad Autónoma del Estado de México, Unidad Académica Profesional Huehuetoca, Academia de Trabajo Social. Línea de Investigación en Gobernanza de la Salud Pública.

cgarciali@uaemex.mx

ORCID: http://orcidd.org/0000-0002-9364-6796 